\title{
Impact of pharmacist discharge counseling on hospital readmission and emergency department visit
}

\author{
Tu Tran*1, Saijal Khattar ${ }^{2}$, Tiffany T. Vu², Maggie Potter ${ }^{1}$, Jane Hodding ${ }^{1}$, Grace M. Kuo ${ }^{2,3}$, Jennifer Le ${ }^{1,2}$ \\ ${ }^{1}$ Department of Inpatient Pharmacy, Long Beach Memorial Hospital, Long Beach, CA, United States \\ ${ }^{2}$ University of California San Diego, Skaggs School of Pharmacy and Pharmaceutical Sciences, La Jolla, CA, United States \\ ${ }^{3}$ University of California San Diego, School of Medicine, Department of Family Medicine and Public Health, La Jolla, CA, \\ United States
}

Received: December 14, 2016

Accepted: January 21, 2017

Online Published: March 13, 2017

DOI: $10.5430 /$ jha.v6n2p68

URL: https://doi.org/10.5430/jha.v6n2p68

\begin{abstract}
Objective: The enactment of the Affordable Care Act (ACA) in 2010 imposes payment penalty on hospitals with high hospital readmission rates. In an effort to reduce readmissions, a pharmacist discharge counseling program was implemented to facilitate transition of care to the outpatient setting. Our study objective was to evaluate the impact of the program on hospital readmissions and visits to the emergency department (ED).

Methods: This was a single-center, retrospective cohort study conducted at a not-for-profit, teaching community hospital with 462 total beds. Pharmacists provided counseling to patients discharged from the medicine floor between November 2013 and January 2014, and included those considered to be high-risk (e.g., taking $\geq 5$ scheduled medications and had diseases such as congestive heart failure and diabetes mellitus). Descriptive analysis was performed and outcomes were compared between patients who did and did not receive pharmacist counseling.

Results: Of a total of 889 discharged patients, 488 (55\%) received counseling from a pharmacist. For the entire cohort, mean age was $55 \pm 20$ years; Charlson Comorbidity Index (CCI) score was $2.74 \pm 2.95$; and length of hospitalization was $4 \pm 4$ days. These parameters were not statistically different between the two groups. Within 30-days after hospital discharge, significantly fewer subjects who received counseling, compared with those who did not, were readmitted to the hospital (11.3\% vs. $15 \%$, $p=.009)$ or visited the $\mathrm{ED}(10.6 \%$ vs. $15 \%, p=.005)$.

Conclusions: Discharge counseling provided by pharmacists during transitions of care at a community hospital significantly reduced 30-day readmission and ED visit rates.
\end{abstract}

Key Words: Transition of care, Discharge pharmacist, Pharmacist counseling, Hospital readmission, Emergency department visit

\section{INTRODUCTION}

Transitions of care is the process of ensuring continuity of healthcare as the patient transfers between different locations and levels of care such as being admitted to a hospital, transferring to a skilled nursing facility, or at the point of being discharged home. At the hospital, patients may be at risk for prescribing errors, dispensing errors, monitoring errors, administering errors, and experiencing adverse events due to lack of communication and inaccurate medication histories taken upon admission. An estimated $60 \%$ of medication errors occur during times of transition, and even 19\% of discharged patients experience an adverse event within

*Correspondence: Tu Tran; Email: ttran11@memorialcare.org; Address: Pharmacy Supervisor, IV Room and PT Lab, United States. 
three weeks post discharge. ${ }^{[1]}$ With the high quality of care received during hospitalization, it is imperative that patients continue to effectively manage their health to prevent hospital readmissions and emergency department (ED) visits.

In order to ensure effective transitions of care, the Affordable Care Act (ACA) in 2010 was established as an effort to reduce hospital readmission. ${ }^{[2]}$ The ACA imposed payment penalties on hospitals with high readmission rates for certain conditions, such as congestive heart failure, pneumonia, and acute myocardial infarction. In 2013, these penalties added up to approximately $\$ 280$ million spanning across 2,200 U.S. hospitals. ${ }^{[3]}$ Common causes of re-hospitalization include lack of patient education, inappropriate medication reconciliation, lack of adherence, and failure to maintain physician visits after discharge. ${ }^{[1]}$ As a result, a community hospital proactively implemented transitions of care services utilizing a pharmacist discharge counseling program in an effort to effectively facilitate the transition of care from inpatient to outpatient settings. This program was established with the intentions to: (1) reduce readmission rates and ED visits and (2) improve patients' quality of life. Our study objective was to evaluate the impact of the counseling program on hospital readmission and visit to the ED.

\section{METHODS}

This was a single-center, retrospective cohort study conducted at Long Beach Memorial Hospital, a not-for-profit, teaching community hospital with 462 total beds. This study was approved by the institutional review boards with the use of a waiver of informed consent.

The pharmacist discharge counseling program was implemented in July 2013. This program incorporated follow-up phone calls and education on managing their disease state and discharge medications to optimize medication therapy. One full-time equivalent pharmacist was employed to implement this program on one medicine floor of the hospital. Patients who were targeted for this program included those with disease states and conditions that placed the patient at increased risk for sub-optimal healthcare services after hospital discharge. These risk factors, or criteria for pharmacist counseling included: (1) concurrent use of $>5$ scheduled medications, (2) end-stage renal disease (ESRD), (3) pneumonia, (4) chronic obstructive pulmonary disease (COPD), (5) diabetes, (6) chronic heart failure (CHF), (7) myocardial infarction, and (8) use of high-alert medications, such as warfarin and insulin.

Table 1. Types of pharmacist interventions at discharge

\begin{tabular}{ll}
\hline Category & Example \\
\hline Therapeutic & - Recommending a different drug that may be more effective based on a patient's profile \\
Recommendation & - Recommending labs to help monitor drug levels and adverse drug reactions \\
Dose Optimization & - Changing a drug dose based on a patient's individual therapeutic and side effect profiles \\
& - Answering patient questions about their medications \\
Facilitate Discharge Process & - Ensure patient has all belongings and patient education materials necessary to continue proper \\
& - Ede outpatient \\
Patient Reinforcement & - Recommending tips that will help a patient remember to take medications on time \\
Preventing Adverse Drug & - Identifying drug-drug interactions \\
Reactions & - Recommending an alternative to a drug that a patient is allergic to \\
Duplicate Therapy & - Assuring that a patient is not on multiple medications that have the same mechanisms of action \\
\hline
\end{tabular}

Demographic and outcomes data were extracted using electronic medical records of subjects counseled between November 2013 and January 2014. Hospital readmission and ED visits, both with discharge diagnoses, were evaluated at postdischarge 1, 2, 3, and 6 months. In addition, the types of pharmacist intervention upon discharge (see Table 1), and follow-up phone call or clinic visit were recorded.

The Charlson Comorbidity Index (CCI) was used to assess for the severity of illness between patients who did and did not receive pharmacist discharge counseling. ${ }^{[4]}$ The CCI is a prognostic point-based scoring system that measures comorbid diseases (e.g., congestive heart failure, dementia, diabetes, leukemia, and acquired immune deficiency syndrome) and their risks for mortality. ${ }^{[5]}$ The summation of individual scores provides the CCI, in which a higher value predicts a greater likelihood of 10-year mortality.

For analysis, patients were divided into two groups: those who received discharge counseling from a pharmacist (Group A) versus those who did not receive discharge counseling from a pharmacist (Group B). Descriptive analysis was performed and outcomes of subjects who received discharge counseling from a pharmacist were compared with those 
who did not receive counseling. Data were analyzed between the two groups using appropriate tests (i.e., student $t$-test for continuous variables, and Chi-square [or Fisher exact] for categorical variables). A significance level of .05 for a two-sided test was assumed when calculating results. All descriptive and statistical analyses were performed using $\mathrm{R}$ version 3.0.1.

\section{RESUlts}

A total of 889 patient records were evaluated, with $488(55 \%)$ patients receiving counseling from a pharmacist. The entire study cohort had an average age of $55 \pm 20$ years; CCI score, $2.74 \pm 2.95$; and length of hospitalization, $4 \pm 4$ days. These parameters were similar between the two groups (see Table 2).

Table 2. Patient demographic data

\begin{tabular}{llll}
\hline Variable & $\begin{array}{l}\text { All patients } \\
(\mathbf{N}=\mathbf{8 8 9})\end{array}$ & $\begin{array}{l}\text { Received Pharmacist Discharge } \\
\text { Counseling }(\mathbf{n}=\mathbf{4 8 8})\end{array}$ & $\begin{array}{l}\text { Not Received Pharmacist } \\
\text { Discharge Counseling }(\mathbf{n}=\mathbf{4 0 1})\end{array}$ \\
\hline No. $(\%)$ of Males & $372(41.8 \%)$ & $210(43 \%)$ & $162(40.4 \%)$ \\
Mean age, years $( \pm S D)$ & $55( \pm 20)$ & $52.7( \pm 20.5)$ & $57.9( \pm 20.6)$ \\
Mean length of Stay, days $( \pm S D)$ & $4( \pm 4)$ & $4( \pm 3.5)$ & $4.2( \pm 5.2)$ \\
Mean Charlson Comorbidity & $2.74( \pm 2.95)$ & $2.55( \pm 2.78)$ & $2.95( \pm 3.13)$ \\
Index $(\mathrm{CCI})$ Score $( \pm S D)$ & & \\
\hline
\end{tabular}

Note. $P$-values for all variables were not statistically significant at $>.05$

The distribution of achieving the criteria for pharmacist counseling varied between Groups A and B. Although everyone in Group A received counseling, 337 (69.1\%) met at last one of the criteria outlined in the methods section to receive counseling. Some patients $(40 \%)$ qualified because they had $\geq 5$ scheduled medications, and the remainder qualified because they received one of the following diagnoses: diabetes mellitus, pneumonia, COPD, ESRD, CHF, or acute myocardial infarction. Approximately $1.6 \%$ of patients also qualified to receive counseling because they were prescribed high-alert medications such as warfarin and insulin that required careful monitoring. While Group B did not receive counseling, 288 patients $(71.8 \%)$ in this group met at least one of the criteria for discharge counseling but counseling was not possible due to inadequate pharmacist time and outside of hours discharges. Approximately $44 \%$ of patients received $\geq 5$ scheduled medications.

After determining which patients received counseling, Group A's patient records were further evaluated to determine if there was any pharmacist intervention in addition to general medication counseling that benefited the patient's treatment. More specifically, the intervention types we observed included therapeutic recommendations, dose optimization, facilitation of the discharge process, patient reinforcement, prevention of adverse drug reactions, prevention of duplication of therapy, and any other interventions that the pharmacist described in the chart notes. Approximately 370 of the $488(75.8 \%)$ counseled patients had pharmacist intervention upon discharge. Furthermore, 187 of the 488 (38.3\%) counseled patients also received a follow-up phone call from a pharmacist to ascertain their health status and to confirm an appointment with their doctors for a follow-up visit after hospital discharge. The follow-up phone calls verified that 151 patients $(30.9 \%)$ had a post-discharge appointment with their doctors.

Each patient's ED visit and/or hospital readmission were recorded from the following post-discharge time points: 30-, 60-, 90-, and 120-days (see Figure 1). Hospital readmissions 30 days after discharge were significantly lower for patients in Group A than Group B (11.3\% vs. 15\%, $p=.009)$. The ED visits 30-days post-discharge were also significantly lower for patients in Group A than Group B (10.6\% vs. 15\%, $p=.005)$. At the 60-day post-discharge, similar number of patients from group A, compared with group B, visited the ED $(14.9 \%$ vs. $17 \%, p=.124)$ and were readmitted to the hospital (18.9\% vs. 19.5\%, $p=.062)$. In addition, Group A patients experienced similar hospital readmissions $(28.1 \%$ vs. $29.7 \%, p=.152)$ and lower ED visits (19.4\% vs. $21 \%$, $p=.235$ ) than Group B at 90-days post-discharge. At 120days post-discharge, Group A, as compared with Group B, had similar readmission and ED visit rates $(36.5 \%$ vs. $36.7 \%$ $(p=.269)$ and $23.1 \%$ vs. $24.5 \%(p=.558)$, respectively.

The patients' admitting diagnoses for their ED visits and hospital readmissions were recorded and compared to their discharge diagnoses from their primary hospitalization (see Figure 2). Of the 156 counseled patients (Group A) who visited the ED or were readmitted within 120-days after discharge, $63(40.4 \%)$ patients received the same diagnoses as their primary hospitalization. Of the 135 Group B patients who visited the ED or were readmitted, 61 (45.2\%) received the same diagnoses. Thus, a higher percentage of Group A patients had different admitting diagnoses from their primary discharge diagnoses when compared with Group B patients $(59.6 \%$ vs. $54.8 \%, p=.478)$

ISSN 1927-6990 E-ISSN 1927-7008 


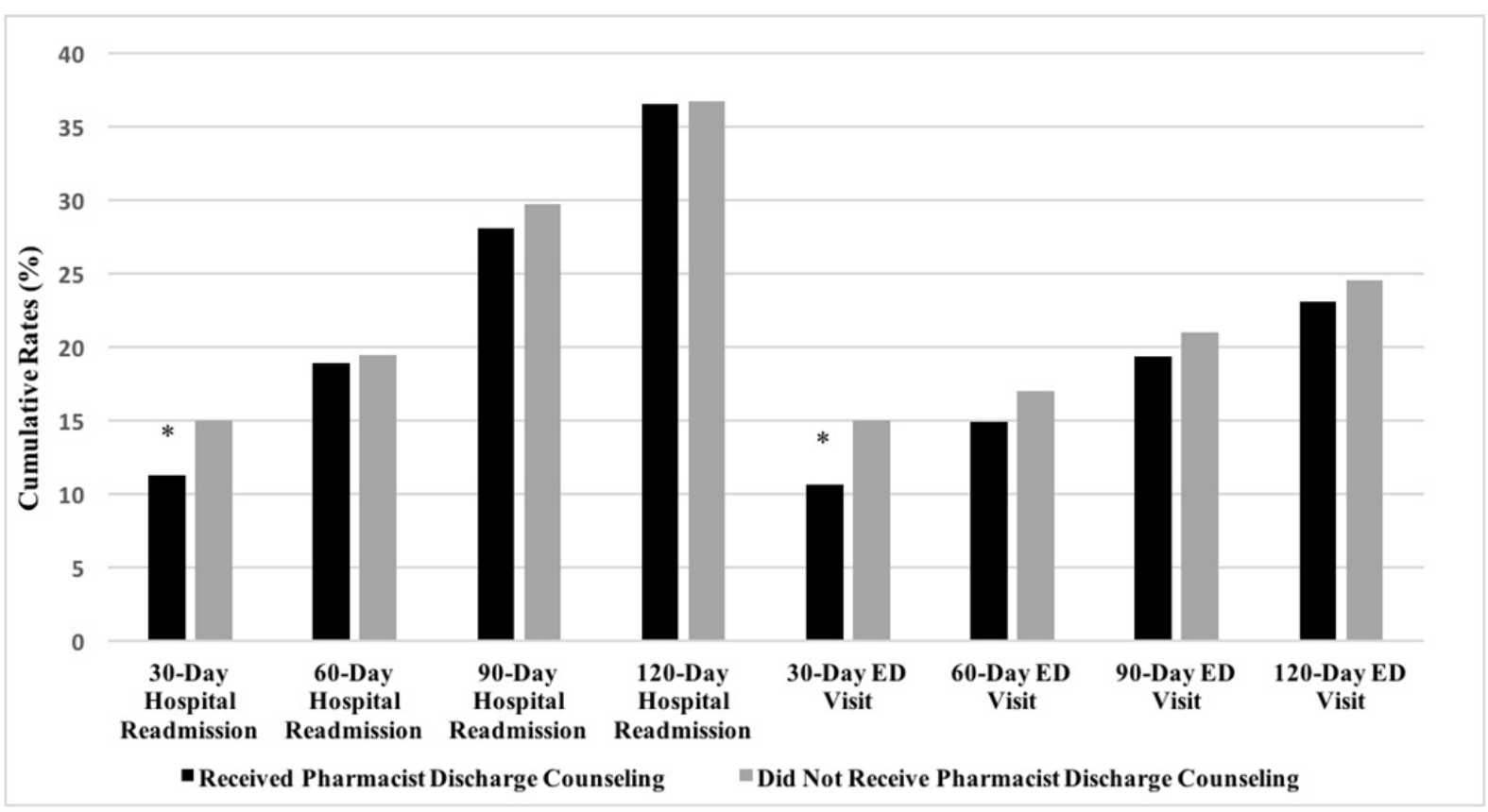

Figure 1. Hospital readmission and ED visit rates post-discharge

${ }^{*}$ Denotes p-value $<.05$

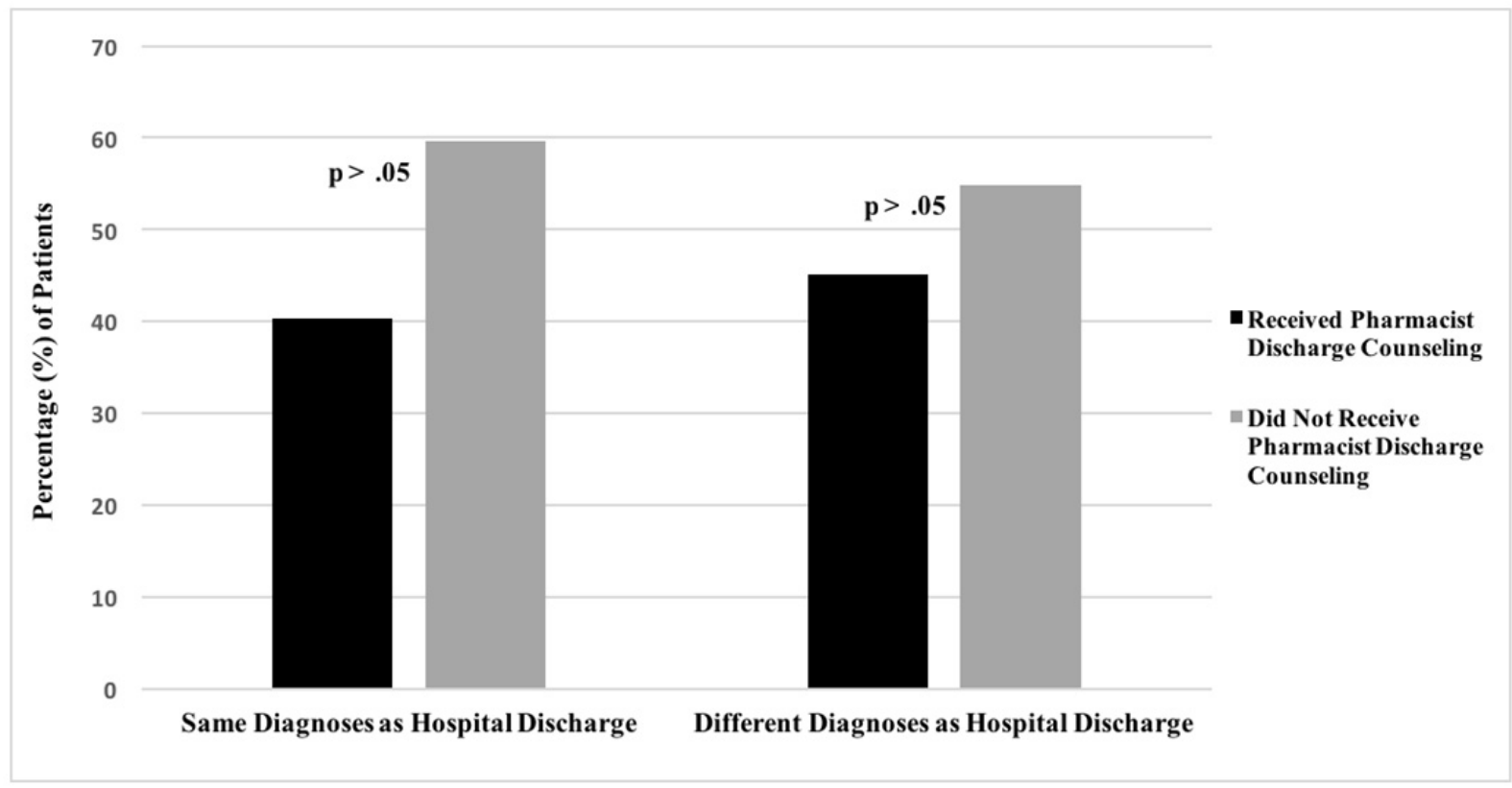

Figure 2. Similarity in admitting diagnoses at hospital readmission or ED visit post-discharge

\section{Discussion}

For this retrospective study, the entire cohort was divided into two groups based on one factor-whether a patient received counseling or not from a pharmacist upon discharge. Most factors that characterized each group such as age, length of stay, and the CCI score were not statistically different between the two groups. In particular, the CCI score was included in our analysis because it is a prognostic indicator of admission and plays a role in improving discharge decisions to prevent readmission rates. CCI has been applied in studies to determine its validity, such as in patients with acute coronary syndrome (ACS) and those who revisited the ED within 72 hours. In patients with ACS, it was determined that comorbidities of nine disease states such as heart failure, diabetes, metastatic tumors, and renal diseases were indicators of increasing hospital mortality. ${ }^{[6]}$ Thus, $\mathrm{CCI}$ is imperative to 
assess patients' state of health upon admission and discharge to determine if this leads to higher rates of readmission.

Over $75 \%$ of patients who were counseled received a valuable interaction with the pharmacist that went beyond a simple medication counseling session. For example, a randomized trial was conducted at a large teaching hospital in Boston to assess the impact of pharmacist interventions at discharge and post-discharge, particularly the impact on the rate of preventable adverse drug events (ADEs) ${ }^{[7]}$ In the intervention group, the pharmacists conducted medication reconciliation at discharge, suggested changes to medications to simplify a medical regimen and improve compliance, to avoid drug interactions, and to reduce possible side effects. They also addressed discrepancies (i.e. dose or frequency) between the discharge medication list and the patient's reported home medication regimen during the follow-up phone call after discharge. At thirty days post-discharge, preventable ADEs occurred in 1 patient in the intervention group and in 8 patients in the usual-care group ( $1 \%$ vs. $11 \% ; p=.01)$. The rate of preventable, medication-related ED visits or hospital readmissions was $1 \%$ in the intervention group and $8 \%$ in the usual-care group $(p=.03)$. As a result, the study concluded that pharmacist interventions at discharge and post-discharge were associated with lower rates of preventable ADEs and ED visits/hospital readmissions 30 days after hospital discharge. ${ }^{[7]}$ Similarly, in our study program, the pharmacist facilitated the discharge process by providing the patients an opportunity to improve their understanding for the purpose their medications and addressing their concerns to improve adherence and proper use, which is critical especially after an acute care encounter. Furthermore, the pharmacist improved the patients' treatment plans by making interventions and, if necessary, recommending changes to a medication regimen after in-depth review of the patients' profiles.

In addition to medication counseling and improving the treatment plan, the discharge pharmacist conducted follow-up phone calls to facilitate patients' recovery post-discharge by ensuring the appropriate use of prescribed medications and confirming a scheduled appointment with their doctors. Such an intervention was also utilized in a randomized, prospective, open clinical trial conducted in Barcelona for patients admitted for heart failure to analyze its impact on treatment adherence. ${ }^{[8]}$ The patients in the intervention group received verbal and written information on the disease, proper diet, and their drug therapy the day of hospital discharge, and follow-up phone calls were made by the pharmacist on a monthly-basis during the first 6 months and every 2 months thereafter for another 6 months to address any medicationrelated problems and clarify any other patient questions. The control group received "the standard care of the center". ${ }^{[8]}$
Patient treatment compliance was defined as reliable if patients were taking $95 \%-100 \%$ of prescribed doses, partially reliable if taking $85 \%-95 \%$ of doses, and non-reliable if taking $<85 \%$ of doses. Patient treatment compliance was higher for the intervention group at 2 months ( $88.2 \%$ vs. $60.5 \% ; p=$ $.002)$, at 6 months ( $91.1 \%$ vs. $69 \% ; p=.015)$, and 12 months ( $85 \%$ vs. $73.9 \% ; p>.05$ ). Additionally, less patients had hospital readmission in the intervention group at 2 months post-discharge $(11.4 \%$ vs. $25 \% ; p=.041)$ and at 6 months ( $24.3 \%$ vs. $42.2 \% ; p=.028)$. Hence, follow-up phone calls are an important step in patient care that can foster adherence to the treatment plan and prevent further hospitalizations. The involvement of a pharmacist in the transition of care contributes to effective care in the outpatient setting where access to a pharmacist with complete understanding of the patient's acute care episode may be hindered.

The 30-day hospital readmission rate is a rising concern across the United States. A main reason behind this high rate has been identified as a "lack of coordination" during transitions such as hospital discharge. ${ }^{[1]}$ In this study, the 30 day post-discharge ED visit and hospital readmission were significantly reduced in patients with pharmacist counseling at hospital discharge. In fact, approximately $4 \%-5 \%$ fewer patients who received pharmacist counseling, as compared with those who did not, visited the ED or readmitted to the hospital within 30 days after initial hospitalization. This study demonstrated that this difference may result from pharmacist counseling; but it is uncertain how dependent this difference is on the various pharmacist interventions and the follow-up phone calls. Each type of intervention requires further analysis and must be compared to a group that did not receive that intervention. Interestingly, the admitting diagnoses for ED visit or hospital readmission in patients who received pharmacist counseling were readmitted or visited the ED for the same diagnoses as their primary hospital stay, but no statistically significant association was found between pharmacist counseling and reduced hospital visits for the same reason.

All patients were assessed under the same criteria in order to qualify for counseling from a discharge pharmacist. Yet, approximately $72 \%$ of the patients who did not receive counseling (Group B) did in fact qualify for pharmacist counseling according to the criteria. Further analysis of these patients' diagnoses and number of comorbid diseases is necessary to definitively understand why they were not prioritized for pharmacist counseling. However, we believe these missed opportunities resulted from the limited availability of pharmacist. At the initiation of our service, only one pharmacist was available for discharge education for the entire medicine floor. There was consistent lapse in this service on weekday 
evenings and weekends. This was one of the major limitations of this study because these patients span a time period of three months during which time the criteria for inclusion into the intervention group evolved and were refined to encompass patients who most likely benefit from this pharmacy service.

Initially only one full-time equivalent pharmacist provided discharge counseling for four days a week. Through November of 2013, another pharmacist was added, increasing discharge counseling to five days a week. The yearly cost of one full-time equivalent pharmacist is approximately $\$ 175,000$ $(\$ 14,500$ per month) plus benefits and the average cost of a 30-day readmission for patients aged 65 years and above was $\$ 13,800$ in 2013. ${ }^{[9]}$ With an estimated $4 \%-5 \%$ fewer patients (6) that were readmitted 30 days post-discharge over the 3-month course of this study, the potential cost savings from penalties add up to $\$ 82,800$ ( $\$ 27,600$ per month). Based on our study findings, the program was expanded to provide discharge counseling for 7 days a week. Furthermore, this discharge pharmacist counseling service has expanded to other floors within the adult hospital and newly implemented in our adjacent pediatric hospital.

Our study results prompting subsequent programmatic expansion demonstrate promise for other hospitals to adopt this pharmacy service to facilitate the transition of care process. Prior to any further expansion or implementation of new programs on other floors or at other hospitals, additional analysis to measure the impact of discharge counseling and pharmacist interventions on patient adherence to medications can

\section{REFERENCES}

[1] Improving Transitions of Care - NTOCC [Internet]. National Transitions of Care Colition; 2008 [cited 2015 Sep 20]. Available from: http://www.ntocc.org/portals/0/pdf/resour ces/policypaper.pdf

[2] Orszag PR, Emanuel EJ. Health Care Reform and Cost Control. New England Journal of Medicine. 2010 Dec; 363(7): 601-3. https://doi.org/10.1056/NEJMp1006571

[3] Joynt KE, Jha AK. A Path Forward on Medicare Readmissions. New England Journal of Medicine. 2013 Mar; 368: 1175-77. https: //doi.org/10.1056/NEJMp1300122

[4] Hines AL, Barrett ML, Jiang HJ, et al. Conditions With the Largest Number of Adult Hospital Readmissions by Payer, 2011 - Statistical Brief \#172 [Internet]. Healthcare Cost and Utilization Project. Agency for Healthcare Research and Quality. 2014 [cited 2015 Sep 20]. Available from: http://www.hcup-us.ahrq.gov/reports/statbrief s/sb172-conditions-readmissions-payer.jsp

[5] Deyo R. Adapting a clinical comorbidity index for use with ICD9-CM administrative databases. Journal of Clinical Epidemiology. provide more insight into the strength of correlation between pharmacist interventions at discharge and patient outcomes. A study could also be conducted in a larger population size with a prospective, randomized trial design to obtain more statistically significant data beyond the 30-day time point after discharge, giving more reliable information on the longterm impact of pharmacist interventions at hospital discharge on readmissions and other patient outcomes.

\section{Conclusions}

More than half the patients who met the study criteria received pharmacist counseling upon discharge from the hospital, and the pharmacist was able to improve a patient's treatment plan through various interventions (i.e., therapeutic recommendations, dose optimization, facilitation of the discharge process, patient reinforcement, etc.) for most patients. Those who received pharmacist counseling had significantly lower readmission and ED visit rates 30 days after hospital discharge compared to those who did not receive pharmacist counseling.

\section{ACKNOWLEDGEMENTS}

This work was presented at the 2015 Seminar of the California Society of Health-Systems Pharmacists in San Diego, CA. This study was not supported by any funding agency. The authors are solely responsible for the study design, data collection and analysis, decision to publish, or preparation of the manuscript.

\section{CONFLicts OF InTEREST Disclosure}

The authors declare they have no conflict of interest.
1992; 45(6): 613-9. https://doi.org/10.1016/0895-4356(92 ) $90133-8$

[6] Radovanovic D, Seifert B, Urban P, et al. Validity of Charlson Comorbidity Index in patients hospitalised with acute coronary syndrome. Insights from the nationwide AMIS Plus registry 2002-2012. Heart. 2013 Jan; 100(4): 288-94. https://doi.org/10.1136/heartj nl-2013-304588

[7] Schnipper JL, Kirwin JL, Cotugno MC, et al. Role of Pharmacist Counseling in Preventing Adverse Drug Events After Hospitalization. Archives of Internal Medicine. 2006; 166(5): 565. https://doi.org/10.1001/archinte.166.5.565

[8] Cabezas CL, Salvador CF, Quadrada DC, et al. Randomized clinical trial of a postdischarge pharmaceutical care program vs. regular follow-up in patients with heart failure. Farmacia Hospitalaria. 2006; 30(6): 328-42. https://doi.org/10.1016/S1130-6343(06) 7 4004-1

[9] Barrett ML, Wier LM, Jiang HJ, et al. All-Cause Readmissions by Payer and Age, 2009-2013. HCUP Statistical Brief \#199. Agency for Healthcare Research and Quality. December 2015. Available from: http://www.hcupus.ahrq.gov/reports/statbriefs /sb199-Readmissions-Payer-Age.pdf 